\title{
B3-lesions of the breast and cancer risk - an analysis of mammography screening patients
}

\author{
OLIVER HOFFMANN ${ }^{1}$, GESINA ATHINA STAMATIS ${ }^{1,2}$, ANN-KATHRIN BITTNER ${ }^{1}$, GEORG ARNOLD ${ }^{3}$, \\ ROLF SCHNABEL $^{4}$, KARLGEORG KRÜGER ${ }^{5}$, RAINER KIMMIG ${ }^{1}$ and MARTIN HEUBNER ${ }^{1}$ \\ ${ }^{1}$ Department of Gynecology and Obstetrics, University Hospital Essen, University of Duisburg-Essen, D-45122 Essen, \\ Germany; ${ }^{2}$ Intensive Care Unit (Critical Care Complex), North Middlesex University Hospital, London N181QX, UK; \\ ${ }^{3}$ Center of Pathology Essen-Mitte; ${ }^{4}$ Department of Pathology Essen-Steele, D-45276 Essen; \\ ${ }^{5}$ Diavero Breast Diagnosis Center, D-45257 Essen, Germany
}

Received September 8, 2015; Accepted January 20, 2016

DOI: $10.3892 / \mathrm{mco} .2016 .790$

\begin{abstract}
The use of mammography screening, followed by needle core biopsy (NCB), is associated with an increasing amount of invasive procedures. A considerable amount of specimens must be classified as lesions with uncertain malignant potential (B3-lesion). In these cases, an open biopsy is indicated for further diagnosis. We evaluated patients with B3-lesions to determine the risk of malignancy corresponding to the histopathological NCB results and the type of radiological lesion identified. A total of 95 patients participating in the German mammography screening program with a B3-lesion following NCB (104 B3-lesions in total) were included in our analysis. We analyzed the correlation between the initial histopathological findings from the NCB specimen and cancer risk. We further analyzed the correlations of malignant results with the type of mammographic lesion. In 23 cases (22\%), histopathological examination following excision revealed a malignant lesion, including invasive and in situ carcinoma. The positive predictive value of the subgroups of B3-lesions ranged between 0.11 and 0.31 ; the $\mathrm{B} 3$-lesion associated with the highest cancer risk was the atypical ductal hyperplasia; however, no significant difference was observed between the B3-lesion subgroups $(\mathrm{P}=0.309)$ regarding the risk of malignancy. Comparing the different types of mammographic findings, such as radiological mass or microcalcifications, there was no significant difference in the risk for malignancy $(\mathrm{P}=0.379)$. The different types of B3-lesions did not exhibit
\end{abstract}

Correspondence to: Dr Oliver Hoffmann, Department of Gynecology and Obstetrics, University Hospital Essen, University of Duisburg-Essen, 55 Hufelandstrasse, D-45122 Essen, Germany E-mail: oliver.hoffmann@uk-essen.de

Abbreviations: ADH, atypical ductal hyperplasia; FEA, flat epithelial atypia; LIN, lobular intraepithelial neoplasia; NCB, needle core biopsy; PL, papillary lesion; PT, phyllodes tumor; RS, radial scar

Key words: breast cancer, mammography screening, B-coding system, B3-lesion, needle core biopsy, open biopsy differences in the risk for malignancy, and the morphological type of mammographic lesion does not appear to be correlated with cancer risk; therefore, our results underline the need for open biopsy in patients with B3-lesions following NCB.

\section{Introduction}

Needle core biopsy (NCB) is an established technique for obtaining tissue samples from breast lesions detected during mammography screening. In the majority of cases, a definitive diagnosis may be reached with this method, without a need for further diagnostic breast surgery. The B-coding system established in the German mammography screening program, employed by the NHS Breast Cancer Screening program, classifies NCBs as follows: B1, normal tissue/non-diagnostic; B2, benign; B3, uncertain malignant potential; B4, suspicious for malignancy; or B5, malignant (1). The majority of NCBs are definitively classified as normal (B1), benign (B2) or malignant (B5) (1). The accuracy of benign and malignant NCB diagnosis is supported by the use of the two borderline categories, B3-lesions (lesions of uncertain malignant potential) and B4-lesions (suspicious for malignancy). The B3 category represents a heterogeneous group of lesions that may be associated with malignant disease and thus require surgical intervention. Even if a benign histopathological diagnosis is made following surgical excision, B3-lesions are linked to an increased risk of associated adjacent malignancy $(1,2)$. Hence, in clinical practice, the majority of cases progress to surgical intervention to establish an excision histological diagnosis. Although the B3 category constitutes a relatively small proportion of all NCBs, namely 5\% (3-6), there are significant implications, particularly for the affected patients, who are usually asymptomatic. With the increasing use of mammographic screening, an increased detection rate of B3-lesions has been observed (3,7), resulting in breast surgery for ultimately benign final histopathological diagnoses. This effect represents a drawback of the mammographic screening system.

Therefore, it is crucial for the use of the B-coding system to be familiar with the precise cancer risk of the patient with a B3-lesion following NCB, in order to be able to offer the patient professional advice. 


\section{Patients and methods}

Patients. We conducted a retrospective analysis of the histopathological results of breast lesions of uncertain malignant potential (B3-lesions). A total of 2,143 patients attended the German Mammography Program of the Screening Unit in Essen, Muelheim and Oberhausen, Germany, between 2006 and 2011, of whom 300 were diagnosed with a B3-lesion. Of those 300 patients 95 provided written informed consent to the evaluation of their data. A total of $104 \mathrm{~B} 3$-lesions were included in our analysis, as some patients had more than one lesion.

Mammography and NCB. Women aged 50-69 years were invited to attend the Breast Screening Program in an interval of 2 years. Each mammogram was reviewed by two independent radiologists. The reason for performing NCB was the presence of a mammographic abnormality. The main mammographic abnormalities were microcalcifications, presence of a mass, and architectural deformity. The NCBs were performed under guidance, either by ultrasound, stereotaxis, or by the vacuum-assisted technique (1). A total of 4 samples of each detected suspicious lesion were collected performing a high-speed biopsy, and 12 samples were collected performing a vacuum-assisted biopsy. The biopsies were reviewed by two independent pathologists. The results of the biopsies were classified as B1 to B5-lesions, according to the UK B-coding guidelines (8). All patients with a B3-lesion on NCB were discussed at a multidisciplinary meeting, attended by breast radiologists, pathologists and gynecologists, and a decision was made regarding further treatment. All patients with uncertain histopathological diagnosis underwent an excision biopsy.

Histopathological diagnosis. Lesions of uncertain malignant potential (B3-lesions) were identified as lobular intraepithelial neoplasia (LIN) grade 1 and 2, atypical ductal hyperplasia (ADH), papillary lesion (PL), flat epithelial atypia (FEA), radial scar (RS) and phyllodes tumor (PT). Our collected data included patient age, mammographic abnormalities, type of core biopsy and imaging technique (i.e., guidance by ultrasound, stereotaxis, or vacuum-assisted biopsy), histopathological result of the NCB, surgical procedure with final diagnosis including histological type, grading and TNM classification and immunohistochemical status when indicated (estrogen and progesterone receptor status and human epidermal growth factor receptor 2 status). To underline the purpose of our study, we categorized the histopathological findings in a group of malignant results, including invasive carcinoma, ductal carcinoma in situ (DCIS) and high-grade LIN (LIN 3), and a group of benign results, including LIN 1 and 2, ADH, PL, FEA, RS and PT. The postsurgical histopathological results were compared with the primary histopathological results of the biopsy and the positive predictive value (PPV) for the detection of a malignant lesion was calculated. PPV was defined as follows: Number of true positives/(number of true positives + number of false positives).

Statistical analysis. We further analyzed whether the risk for malignancy differed between types of B3-lesions using the $\chi^{2}$-test according to Pearson. Furthermore, we analyzed whether the type of radiological abnormality in B3-lesions
Table I. Incidence of the subgroups of B3-lesions, malignant postsurgical results and PPV.

\begin{tabular}{lccr}
\hline B3-lesion & Incidence (\%) & $\begin{array}{c}\text { Malignant diagnosis } \\
\text { after excision }\end{array}$ & PPV \\
\hline LIN & $17(16)$ & 5 & 0.29 \\
ADH & $32(31)$ & 10 & 0.31 \\
PL & $24(24)$ & 2 & 0.08 \\
FEA & $14(13)$ & 2 & 0.14 \\
RS & $15(14)$ & 4 & 0.27 \\
PT & $2(2)$ & 0 & 0 \\
Total & 104 & 23 & 0.22 \\
\hline
\end{tabular}

PPV, positive predictive value; LIN, lobular intraepithelial neoplasia; $\mathrm{ADH}$, atypical ductal hyperplasia; PL, papillary lesion; FEA, flat epithelial atypia; RS, radial scar; PT, phyllodes tumor.

was predictive of the risk for malignancy. Statistical analyses were performed using IBM SPSS 20.0 software for Windows (IBM SPSS, Armonk, NY, USA).

\section{Results}

Postoperative histopathological results. The final postoperative histopathological results following excision of bioptic B3-lesions of 95 patients participating in the mammographic screening program were evaluated to determine the potential cancer risk of breast lesions of uncertain malignant potential (B3-lesions). The corresponding mammograms were classified as Breast Imaging-Reporting and Data System (BI-RADS) 4a (85\%) and 4b (13\%), and 2 mammograms were classified as BI-RADS 5 (2\%). Biopsies of all mammographic abnormalities were collected either by high-speed biopsy (27\%) or vacuum-assisted biopsy (73\%). On subclassifying the B3-lesions, in the majority of the cases ADH was detected (31\%). B3-lesions were further subclassified into PL in $20 \%$, low-grade LIN in $16 \%$, RS in $15 \%$ and FEA in $14 \%$ of the patients. Only 2 patients with a B3-lesion were histologically diagnosed with a PT (2\%). To evaluate the diagnostic value of B3-lesions, the initial histology report was compared with the histological results following excision of the lesions.

In 23 cases (22\%), postsurgical histological examination revealed a malignant pathology, including invasive and in situ carcinoma (DCIS, high-grade LIN).

B3-lesion subgroups, malignant postsurgical results and PPV. ADH represented the largest subgroup of B3-lesions and had the highest malignancy rate, with a PPV of 0.31. LIN and RS in the biopsy specimen exhibited an increased malignancy risk in our analysis, with a PPV of 0.29 and 0.25 , respectively. FEA had a PPV of 0.14. PL, representing the second biggest subgroup of B3-lesions, was associated with the lowest risk for malignancy, with a PPV of 0.11. PT was detected in only 2 cases by NCB; in both cases, the initial and final histopathological results matched. The incidence of malignant postsurgical results and the respective PPVs of the subgroups of B3-lesions are presented in Table I. 
Table II. Distribution of the postsurgical histopathological results according to the B3-lesion subgroups.

\begin{tabular}{lccccc}
\hline Lesion & DCIS & Invasive ductal cancer & Grade 3 LIN & Invasive lobular cancer & Number of lesions (\%) \\
\hline LIN & 2 & 0 & 2 & 1 & $5 / 17(29)$ \\
ADH & 7 & 2 & 1 & 0 & $10 / 32(31)$ \\
PL & 2 & 0 & 0 & 0 & $2 / 24(8)$ \\
FEA & 1 & 1 & 0 & 0 & $2 / 14(14)$ \\
RS & 3 & 1 & 0 & 0 & $4 / 15(27)$ \\
PT & 0 & 0 & 0 & 0 & $0 / 2$ \\
Total & 15 & 4 & 3 & 1 & $23 / 104(22)$ \\
\hline
\end{tabular}

DCIS, ductal carcinoma in situ; PPV, positive predictive value; LIN, lobular intraepithelial neoplasia; ADH, atypical ductal hyperplasia; PL, papillary lesion; FEA, flat epithelial atypia; RS, radial scar; PT, phyllodes tumor.

Table III. Detected mammographic lesions according to B3-lesion subgroups.

\begin{tabular}{lcccc}
\hline Lesion & Mass $(\%)$ & Microcalcifications $(\%)$ & Microcalcifications and mass $(\%)$ & Others $(\%)$ \\
\hline LIN & $2(12)$ & $14(82)$ & $1(6)$ & 0 \\
ADH & $5(16)$ & $22(69)$ & $5(16)$ & 0 \\
PL & $2(8)$ & $10(42)$ & $2(8)$ & 0 \\
FEA & $1(7)$ & $8(57)$ & $2(36)$ & $1(7)$ \\
RS & $8(53)$ & $4(27)$ & 0 & 0 \\
PT & $2(100)$ & 0 & $15(14)$ & $3(3)$ \\
Total & $28(27)$ & $58(56)$ & & \\
\hline
\end{tabular}

PPV, positive predictive value; LIN, lobular intraepithelial neoplasia; ADH, atypical ductal hyperplasia; PL, papillary lesion; FEA, flat epithelial atypia; RS, radial scar; PT, phyllodes tumor.

Table IV. Distribution of malignant histopathological results according to the mammographic findings.

\begin{tabular}{lcrr}
\hline Mammographic finding & Incidence & Malignant diagnosis after excision & PPV \\
\hline Microcalcifications & 58 & 14 & 0.24 \\
Mass & 28 & 4 & 0.14 \\
Microcalcifications + mass & 15 & 5 & 0.33 \\
Others & 3 & 0 & 0 \\
\hline
\end{tabular}

As regards the risk for malignancy, no significant difference was observed between the $\mathrm{B} 3$-subgroups $(\mathrm{P}=0.309)$. Detailed information on the postsurgical results according to the B3-subgroups are presented in Table II.

Mammographic abnormalities. B3-lesions exhibited different mammographic abnormalities. On mammography, microcalcifications were detected in 58 of the 104 cases $(56 \%)$; 28 patients $(27 \%)$ were diagnosed with a mass; 15 patients (14\%) exhibited microcalcifications as well as a mass; and 3 patients (3\%) had neither a mass nor microcalcifications, but exhibited other abnormalities, such as an architectural deformity. LIN, ADH and FEA were predominantly associated with microcalcifications. Both cases of PT presented as a mass on mammography. Detailed information on the radiological findings is provided in Table III.

When comparing the different mammographic findings, there was no significant difference in the risk for malignancy $(\mathrm{P}=0.379)$ (Table IV).

\section{Discussion}

B3-lesions are a heterogeneous group of breast lesions. Their detection has increased due to screening mammography. In this study we evaluated the data of 2,143 patients participating in a mammography screening program between 2006 and 2011. A total of $14 \%(n=300)$ of the lesions were B3-lesions (lesions of uncertain malignant potential) and 95 patients were 
reviewed in this study. The rate of B3-lesions in other trials varies between 5 and $18 \%(4,9-12)$.

In our trial, ADH was the biggest subgroup of the B3-lesions, with 31\%; similar observations have been made by other authors (5). The incidence of the other subgroups, such as PT, LIN, FEA and RS, differ among previous trials (4,9-10,13). Bianchi et al (14) demonstrated in a trial with 104 non-malignant papillary lesions (B3) that the underestimation rate for B3-lesions with epithelial atypia was $47.8 \%$, whereas the underestimation rate for B3-lesions without epithelial atypia was $13.2 \%$. B3-lesions were classified as BI-RADS 4 or 5 on mammography in our trial. According to the literature, the malignancy rate for BI-RADS 4 is $\sim 20-30 \%$ and for BI-RADS 5 $\sim 95 \%$ (3,15-17). The overall risk for malignancy of B3-lesions was $22 \%$ in our study; in 23 cases, postsurgical histological examination revealed a malignancy. Other studies reported an incidence of 8-35\% (4-6,13,18-20). The highest malignancy rate regarding the subgroup of the B3-lesions was found for $\mathrm{ADH}$, with a PPV of 0.31 . Our results are in line with the findings of other studies, which did not observe significant differences between different B3-lesions in terms of the risk for malignancy. Our findings confirm the heterogeneity of B3-lesions and their malignant potential. The only NCB result that matched the final diagnosis following excision was PT. However, this tumor accounted for only $2 \%$ of the B3-lesions. Moreover, a PT should be surgically removed in any case, according to the current guidelines. In 23 of the 104 cases (22\%), the histopathological examination following excision revealed a malignant result. The vacuum core biopsy technique is considered to be more sensitive, and it may be hypothesized that its extended use may enable more reliable detection of malignant lesions compared with NCB. However, Londero et al (21) compared the diagnostic techniques and concluded that malignancy rate is underestimated. Although considerably more tissue may be obtained by vacuum biopsy compared with NCB, it cannot replace surgical excision and surgical therapy is recommended in the case of high-risk lesions (ADH and LIN).

In our analysis, the B3-lesion associated with the highest tumor risk was ADH, with a PPV of 0.31. However, different types of B3-lesions did not exhibit significant differences in their risk for malignancy. The morphological type of mammographic lesion does not appear to affect tumor risk. Although the majority of the patients with B3-lesions have a benign final histopathological diagnosis, our results emphasize the indication for surgical excision of the lesions as a diagnostic and therapeutical method in patients with B3-lesions following NCB.

\section{References}

1. Ellis O, Humphreys S, Michell M, Pinder SE, Wells CA and Zakhour HD: Guidelines for non-operative diagnostic procedures and reporting in cancer screening. NHSBSP Publications 50: $35-40,2001$.

2. Rakha EA and Ellis IO: An overview of assessment of prognostic and predictive factors in breast cancer needle core biopsy specimens. J Clin Pathol 60: 1300-1306, 2007.

3. Lee AH, Denley HE, Pinder SE, Ellis IO, Elston CW, Vujovic P, Macmillan RD and Evans AJ; Nottingham Breast Team: Excision biopsy findings of patients with breast needle core biopsies reported as suspicious of malignancy (B4) or lesion of uncertain malignant potential (B3). Histopathology 42: 331-336, 2003.
4. Houssami N, Ciatto S, Bilous M, Vezzosi V and Bianchi S: Borderline breast core needle histology: Predictive values for malignancy in lesions of uncertain malignant potential (B3). Br J Cancer 96: 1253-1257, 2007.

5. Dillon MF, McDermott EW, Hill AD, O'Doherty A, O'Higgins N and Quinn CM: Predictive value of breast lesions of 'uncertain malignant potential' and 'suspicious for malignancy' determined by needle core biopsy. Ann Surg Oncol 14: 704-711, 2007.

6. Maclean GM, Courtney SP, Umeh H, Sanjeev S, McCormick C and Smith BM: Is mode of presentation of B3 breast core biopsies (screen-detected or symptomatic) a distinguishing factor in the final histopathologic result or risk of diagnosis of malignancy? World J Surg 37: 2607-2612, 2013.

7. Liberman L: Clinical management issues in percutaneous core breast biopsy. Radiol Clin North Am 38: 791-807, 2000.

8. Ellis IO, Humphreys S, Michell M, Pinder SE, Wells CA and Zakhour HD; UK National Coordinating Committee for the Breast Screening Pathology; European Commission Working Group on Breast Screening Pathology: Best Practice No 179. Guidelines for breast needle core biopsy handling and reporting in breast screening assessment. J Clin Pathol 57: 897-902, 2004.

9. Lieske B, Ravichandran D, Alvi A, Lawrence DA and Wright DJ: Screen-detected breast lesions with an indeterminate (B3) core needle biopsy should be excised. Eur J Surg Oncol 34: 1293-1298, 2008.

10. Weigel S, Decker T, Korsching E, Biesheuvel C, Wöstmann A, Böcker W, Hungermann D, Roterberg K, Tio J and Heindel W: Minimal invasive biopsy results of 'uncertain malignant potential' in digital mammography screening: High prevalence but also high predictive value for malignancy. Rofo 183: 743-748, 2011.

11. Rajan S, Shaaban AM, Dall BJ and Sharma N: New patient pathway using vacuum-assisted biopsy reduces diagnostic surgery for B3 lesions. Clin Radiol 67: 244-249, 2012.

12. Bernardi D, Borsato G, Pellegrini M, Tuttobene P, Fanto C, Valentini M, Aldovini D and Ciatto S: On the diagnostic accuracy of stereotactic vacuum-assisted biopsy of nonpalpable breast abnormalities. Results in a consecutive series of 769 procedures performed at the Trento department of breast diagnosis. Tumori 98: 113-118, 2012.

13. El-Sayed ME, Rakha EA, Reed J, Lee AH, Evans AJ and Ellis IO: Predictive value of needle core biopsy diagnoses of lesions of uncertain malignant potential (B3) in abnormalities detected by mammographic screening. Histopathology 53: 650-657, 2008.

14. Bianchi S, Bendinelli B, Saladino V, Vezzosi V, Brancato B Nori J and Palli D: Non-malignant breast papillary lesions-B3 diagnosed on ultrasound-guided 14-gauge needle core biopsy: Analysis of 114 cases from a single institution and review of the literature. Pathol Oncol Res 21: 535-546, 2015.

15. Lacquement MA, Mitchell D and Hollingsworth AB: Positive predictive value of the breast imaging reporting and data system. J Am Coll Surg 189: 34-40, 1999.

16. Orel SG, Kay N, Reynolds C and Sullivan DC: BI-RADS categorization as a predictor of malignancy. Radiology 211: 845-850, 1999.

17. Barth V: Atlas der Mammadiagnostik: Mammographie und Sonographie Intensiv Trainieren. 2nd edition. Thieme, Stuttgart, p97, 2006.

18. Tonegutti M, Girardi V, Ciatto S, Manfrin E and Bonetti F: B3 breast lesions determined by vacuum-assisted biopsy: How to reduce the frequency of benign excision biopsies. Radiol Med 115: 1246-1257, 2010 (In English, Italian).

19. de Beça FF, Rasteiro C, Correia A, Costa S and Amendoeira I: Improved malignancy prediction by $\mathrm{B} 3$ breast lesions subclassification. Ann Diagn Pathol 17: 434-436, 2013.

20. Arbeitsgemeinschaft für Gynäkologische Onkologie. http://www. ago-online.de/fileadmin/downloads/leitlinien/mamma/maerz 2014/de/2014D_06_Laesionen_mit_unsicherem_biologischen_ Potenzial_(B3).pdf. Accessed March 27, 2014.

21. Londero V, Zuiani C, Linda A, Battigelli L, Brondani G and Bazzocchi M: Borderline breast lesions: Comparison of malignancy underestimation rates with 14-gauge core needle biopsy versus 11-gauge vacuum-assisted device. Eur Radiol 21: 1200-1206, 2011. 